\title{
Aprendizaje Cooperativo y Portafolio Digital de Grupo: Desarrollo de Competencias
}

\author{
C. Gil ${ }^{1}$, R. Baños ${ }^{2}$, M.G. Montoya ${ }^{1}$, R.I. Herrada ${ }^{3}$, F.G. Montoya ${ }^{4}$ \\ ${ }^{1}$ Dpto. de Informática, ceiA3, Universidad de Almería. Carretera de Sacramento s/n, E-04120 \\ Almería(España), \{cgil,dgil\}@ual.es \\ ${ }^{2}$ Dpto. de Arquitectura y Tecnología de Computadores, CITIC-UGR, Universidad de \\ Granada. C/Periodista Daniel Saucedo s/n, E-18071 Granada (España), rbanos@ugr.es \\ ${ }^{3}$ Dpto. de Didáctica y Organización Escolar, Universidad de Murcia. Campus de Espinardo, \\ E-30100 Murcia (España), rherrada@um.es \\ ${ }^{4}$ Dpto. de Ingeniería. Universidad de Almería. Carretera de Sacramento s/n, E-04120 \\ Almería (España), pagilm@ual.es
}

Resumen. Con el objetivo de determinar la efectividad en el uso del Aprendizaje Cooperativo y el uso del Portafolio Digital de grupo en el proceso de enseñanza-aprendizaje-evaluación, se han analizado los resultados obtenidos en relación al número de alumnos que han superado las diferentes asignaturas en los últimos años de aplicación de estas metodologías. Los resultados muestran que el uso de dichas metodologías conlleva una mayor motivación del estudiante hacia la materia, y un descenso del número de abandonos. Igualmente la motivación del profesorado hacia la enseñanza se ha visto reforzada por la mejora del rendimiento obtenido por la mayoría de los grupos de aprendizaje cooperativo que han desarrollado y completado su portafolio.

Palabras Clave: Aprendizaje Cooperativo, Competencias, Portafolio Digital, Arquitectura de Computadores, TIC.

\begin{abstract}
With the aim of determining the effectiveness of Cooperative Learning in combination with the Group Digital Portfolio in the teachinglearning-assessment process, we have analyzed the results in relation to the number of students who approved the subject in the years in which of applying these methodologies have been applied. The results show that the use of these methodologies involves greater student motivation toward the subject, and a decrease in the number of dropouts. Moreover, lecturers' motivation toward learning has been reinforced by the improvement of performance as most cooperative learning groups who have developed their portfolio.
\end{abstract}

Keywords: Cooperative Learning, Competencies, Digital Portfolio, Computer Architecture, ICT. 


\section{Introducción}

En el contexto actual, el trabajo se organiza cada vez más en equipos multidisciplinares y multiculturales capaces de trabajar en contextos caracterizados por los constantes cambios a los que están sometidos. En estos equipos se valora especialmente la participación, la comunicación, y el trabajo en común de tipo cooperativo. Por ello, resulta necesario que alumnado y profesorado desarrollen la cooperación en las aulas como medio para prepararse para la sociedad actual y del futuro. La primera consecuencia práctica que surge es la necesidad real de adecuar el currículo formativo a las claves de una educación basada en competencias. Sin embargo, el factor fundamental para poder desarrollar dichas competencias consiste en utilizar las metodologías docentes más adecuadas para cada una de ellas, y en cualquier caso deberán ser metodologías activas que impliquen una mayor participación de los estudiantes.

De estas metodologías activas, el Aprendizaje Cooperativo (AC) es uno de los procedimientos de enseñanza-aprendizaje más utilizados por aquellos docentes que desean maximizar el aprendizaje de los estudiantes, y que dicho aprendizaje sea retenido a largo plazo [1], incluso en aquellas materias altamente complejas y difíciles de entender y dominar.

La cooperación consiste en trabajar juntos para alcanzar objetivos comunes. En una situación cooperativa, los individuos procuran obtener resultados que sean beneficiosos para ellos mismos y para todos los demás miembros del grupo. El aprendizaje cooperativo es el empleo didáctico de grupos reducidos en los que los alumnos trabajan juntos para maximizar su propio aprendizaje y el de los demás. Este método contrasta con el aprendizaje individualista y competitivo, en el que los estudiantes trabajan por su cuenta para lograr metas de aprendizaje desvinculadas de las de los demás alumnos [2]. Por ello, es muy importante que el trabajo cooperativo cuente con técnicas de evaluación apropiadas, ya que la evaluación es un modo excelente de hacer que los estudiantes empleen tiempo en sus tareas. Sin embargo, una de las principales dificultades para mejorar la motivación por aprender radica en los modos de evaluación tradicionalmente utilizados por los profesores, normalmente exámenes centrados en la identificación de resultados producidos por el alumno de forma puntual con ocasión de la evaluación [3]. El efecto de estas prácticas es, por lo general, que el alumno estudia para aprobar y no para aprender, actitud que dificulta la comprensión y adquisición de competencias así como el desarrollo de una actitud reflexiva que potencie la capacidad de autorregulación y aprendizaje autónomo. Así, la evaluación constituye uno de los puntos débiles de la docencia universitaria [4].

Pese a ello, existen soluciones que permiten incorporar la autorreflexión, lo que refuerza el aprendizaje del alumno debido a que le proporciona oportunidades para autoevaluar su propio crecimiento. En este sentido, el Portafolio es un método de enseñanza, aprendizaje y evaluación que consiste en la aportación de producciones de diferente índole por parte del estudiante a través de las cuáles se pueden juzgar sus capacidades en el marco de una disciplina o materia de estudio. Estas producciones informan del proceso personal seguido por el estudiante, permitiéndole a él y a los demás ver sus esfuerzos y logros, en relación a los objetivos de aprendizaje y criterios de evaluación establecidos previamente [5]. Más concretamente, el auge de Internet 
ha permitido la extensión en el uso del Portafolio digital como método de enseñanza y aprendizaje, que, gracias a su naturaleza gráfica y habilidad para soportar enlaces entre distintas evidencias digitalizadas, proporciona al alumnado la posibilidad de integrar los aprendizajes de un modo positivo, progresivo y consciente con un gran potencial atractivo [5].

La aplicación práctica del trabajo cooperativo en conjunción con el Portafolio digital de grupo, se ha llevado a cabo en varias asignaturas de Ingeniería Informática así como en el master oficial de Técnicas Informáticas Avanzadas y en el Master de Profesorado de Secundaria, impartidos en la Universidad de Almería. La razón fundamental del cambio metodológico que venimos desarrollando en los últimos años tiene como objetivo final implicar a los estudiantes de forma activa en su proceso de aprendizaje, además de desarrollar las competencias específicas y transversales de cara a su formación integral.

\section{Metodologías de Trabajo en Equipo}

El trabajo en equipo no es sólo un recurso metodológico para enseñar y aprender los contenidos de las distintas áreas, sino también algo que los alumnos deben aprender, como un contenido más, y que, por lo tanto, debe enseñarse de una forma tan sistematizada, al menos, como se enseñan los demás contenidos. Por ello, podríamos suscribir la afirmación que, sobre este aspecto, hacen Johnson y Johnson [6]:

La capacidad de todos los alumnos de aprender a trabajar cooperativamente con los demás es la piedra clave para construir y mantener matrimonios, familias, carreras y amistades estables. Ser capaz de realizar habilidades técnicas como leer, hablar, escuchar, escribir, calcular y resolver problemas es algo valioso pero poco útil si la persona no puede aplicar estas habilidades en una interacción cooperativa con las otras personas en el trabajo, en la familia y en los entornos comunitarios. La manera más lógica de enfatizar el uso del conocimiento y las habilidades de los alumnos dentro de un marco cooperativo, tal como deberán hacer cuando sean miembros adultos de la sociedad, es dedicar mucho tiempo al aprendizaje de estas habilidades en relaciones cooperativas con los demás.

No hay nada mágico en el trabajo en equipo. Algunos tipos de grupos facilitan el aprendizaje de los alumnos y mejoran la calidad de vida en el aula. Otros entorpecen el aprendizaje y provocan insatisfacción y falta de armonía en la clase. Para manejar con eficacia los grupos de aprendizaje, el docente debe saber qué es y qué no es un grupo cooperativo.

\subsection{Aprendizaje Cooperativo}

El objetivo de poner en marcha metodologías activas como Aprendizaje Cooperativo ha sido logar la implicación y participación de todos los alumnos en su propio proceso de aprendizaje a través de objetivos comunes. Se ha desarrollado una estrategia de enseñanza centrada en el alumno que permite 
producir conocimiento desde la interacción en un grupo de aprendizaje cooperativo. También se pretende estimular y desarrollar competencias como el trabajo en equipo, la comunicación oral y la evaluación.

Cooperar significa trabajar juntos para lograr objetivos compartidos. En las actividades cooperativas, los individuos buscan resultados que resulten beneficiosos para sí mismos y, al mismo tiempo, para todos los otros integrantes del grupo. El Aprendizaje Cooperativo consiste en el uso educativo de pequeños grupos que permiten a los estudiantes trabajar juntos para mejorar su propio aprendizaje y el de los demás. Para que los grupos de aprendizaje cooperativo sean realmente eficaces y se acerquen en lo posible a los niveles de mayor rendimiento, deben incorporarse a cada clase cinco elementos básicos de dicho sistema de enseñanza-aprendizaje: la interdependencia positiva, la responsabilidad individual, la interacción cara a cara, la enseñanza de prácticas interpersonales, y la autoevaluación grupal [7].

El aprendizaje cooperativo comprende diferentes grupos de aprendizaje. Por un lado, encontramos a los grupos informales, que se desarrollan en el periodo de una clase. Por otro lado, los grupos formales y los grupos base, que se tienen una mayor duración en el tiempo, en nuestro caso los usaremos indistintamente, y comprenden un cuatrimestre o un curso. Nos centraremos en estos últimos que son los que se han analizado en las asignaturas tratadas. En los grupos formales de aprendizaje cooperativo los estudiantes trabajan juntos para lograr objetivos comunes, asegurándose de que ellos mismos y sus compañeros de grupo completen la tarea de aprendizaje asignada. Cualquier tarea, de cualquier materia y dentro de cualquier programa de estudios, puede organizarse en forma cooperativa. Cualquier requisito del curso puede ser reformulado para adecuarlo al aprendizaje cooperativo formal [2].

Cuando se emplean grupos formales de aprendizaje cooperativo, el docente debe: (a) especificar los objetivos de la clase, (b) tomar una serie de decisiones previas a la enseñanza, (c) explicar la tarea y la interdependencia positiva a los alumnos, (d) supervisar el aprendizaje de los alumnos e intervenir en los grupos para brindar apoyo en la tarea o para mejorar el desempeño interpersonal y grupal de los alumnos, y (e) evaluar el aprendizaje de los estudiantes y ayudarlos a determinar el nivel de eficacia con que funcionó su grupo. Los grupos formales de aprendizaje cooperativo garantizan la participación activa de los alumnos para trabajar el material, explicarlo, resumirlo e integrarlo a las estructuras conceptuales existentes [2].

Es importante que los grupos se formen desde la diversidad de talentos y posibilidades intelectuales [8]. Esto enriquecerá el intercambio y preparará el terreno para el salto cognitivo de los más lentos.

\subsection{Portafolio Digital de Grupo}

El Portafolio puede usarse para el desarrollo y valoración del conocimiento de una asignatura, para la adquisición de habilidades de enseñanza y prácticas reflexivas, así como para la preparación profesional y vocacional. En nuestro caso, el uso del Portafolio digital de grupo en un entorno de aprendizaje cooperativo ha permitido trabajar competencias transversales que difícilmente podríamos desarrollar a través de la clase expositiva tradicional y la posterior evaluación a través del examen final. 
Un Portafolio es una colección de trabajos que incluyen los logros individuales o grupales, tales como resultados de tareas genuinas, la evaluación del proceso, test convencionales o muestras de trabajo. Es decir, documenta los logros alcanzados a lo largo del tiempo. Generalmente el individuo elige el tipo de trabajo que le sirva mejor para expresar su éxito, así como para demostrar su aprendizaje respecto a un objetivo particular como podría ser la certificación o la evaluación tanto sumativa como formativa. De forma genérica, las principales características del Portafolio son las siguientes:

- Es una herramienta reflexiva que permite almacenar y preservar evidencia del crecimiento del alumno, profesor, institución, proyecto, etc.

- Es una colección de trabajos que nos permite conocer lo que el individuo, equipo de trabajo o institución sabe y puede hacer.

- Su contenido es auténtico, con un objetivo representativo y auto-dirigido.

- Constituyen una alternativa para evaluar, certificar, informar, promover, etc.

El Portafolio no es un fin en sí mismo, sino que más bien gracias a él se consigue un aprendizaje continuo y exitoso, el cual se debe a la asociación de la evaluación con las prácticas y procesos de enseñanza llevados a cabo con dicho método [3].

El desarrollo del Portafolio implica documentar, no sólo los logros conseguidos, sino también las autoevaluaciones, las estrategias aplicadas, y el análisis sobre las experiencias de aprendizaje, por lo que es más que una simple colección de tareas.

En la actualidad, las TIC aportan muchas potencialidades a la hora de diseñar y elaborar un Portafolio. En esta línea, las TIC pueden ayudar en la elaboración de las evidencias que conforman el Portafolio y también pueden actuar como plataforma base del proceso de enseñanza y aprendizaje. Una de las principales ventajas del Portafolio digital es que toda la información puede estar disponible para todos los estudiantes y pueden aprender a desarrollar las tareas y actividades aprendiendo con otros compañeros, pueden tutorizar a otros grupos cuando les plantean cuestiones sobre sus actividades, además de poder participar en el proceso de co-evaluación.

\section{Experiencia Práctica en Arquitectura de Computadores}

Como se ha comentado anteriormente, el contexto donde han tenido lugar las experiencias ha sido en las asignaturas de $4^{\circ}$ curso de Ingeniería Informática: Arquitecturas Especializadas y Arquitectura de Computadores, cuya impartición finaliza este año y en las asignaturas de master oficiales como "Optimización Multiobjetivo: Estrategias de Paralelismo” del master oficial en Técnicas Informáticas Avanzadas y "Metodologías Activas de Trabajo en Equipo" del master oficial de Profesorado de Educación Secundaria de la Universidad de Almería.

Aunque las experiencias han sido muy similares, nos centraremos en la asignatura de “Arquitectura de Computadores" de $4^{\circ}$ curso de Ingeniería Informática. Hay que resaltar que la experiencia realizada no ha estado dentro de ningún plan piloto y que, por tanto, se puede considerar como cambios metodológicos en asignaturas de un plan de estudios que se encuentra en proceso de extinción. Esto demuestra que no es 
necesario cambiar un plan de estudios para introducir cambios en la motivación y mejora de resultados de cualquier asignatura.

En algunas de las clases de teoría se ha mezclado la clase expositiva con la resolución de problemas a través de grupos informales de aprendizaje cooperativo creados en cada clase. El mero hecho de hacer un cambio entre una explicación y la realización de un problema hace que los alumnos retomen el control. Discuten por parejas y en algunos casos toda la clase debate sobre posibles soluciones.

En la mayor parte de las sesiones se ha trabajado con grupos formales de aprendizaje cooperativo. Es en estos grupos formales de aprendizaje cooperativo donde centramos nuestra experiencia.

\subsection{Aprender a Trabajar en Equipo en Arquitectura de Computadores}

No aplicar un método que no nos acaba de satisfacer, evidentemente, es muy legítimo. Pero si el trabajo en equipo, además de un método, es también un contenido que deben aprender los alumnos, no podemos eludir su enseñanza diciendo que los alumnos no saben trabajar en equipo, sino que tendremos que enseñárselo, identificando qué es lo que no funciona, cambiando lo que sea necesario, e insistiendo tanto como haga falta hasta que lo aprendan [9]. Este sería el caso además, cuando la competencia transversal "trabajo en equipo" forma parte de las competencias asignadas a una asignatura. En estos años hemos detectado la importancia y la necesidad de enseñar a nuestros alumnos a cooperar y a trabajar en equipo como técnica clave para que el Aprendizaje Cooperativo sea mucho más eficaz y productivo en el aprendizaje y crecimiento integral de nuestros estudiantes. Para ello hemos elaborado un plan de trabajo para enseñar a cooperar y trabajar en equipo, como necesidad previa para el mejor funcionamiento de los grupos cooperativos. Este plan, adaptado de [9,10], incluye distintas actividades y dinámicas para cubrir los siguientes objetivos:

- Mostrar a los alumnos la importancia de saber trabajar en equipo.

- Despertar el interés de los alumnos para trabajar en equipo.

- Enseñar a los alumnos en qué consiste el trabajo en equipos cooperativos y como se puede mejorar.

- Ayudar a los alumnos a organizar su equipo.

- Desarrollar habilidades para la resolución de conflictos.

- Evaluar las actividades de trabajo en equipo.

Una vez que aprendidas y practicadas estas habilidades en una determinada materia, ya estarían en disposición de trabajar en equipo o cooperativamente en cualquier otra asignatura.

\subsection{Trabajar en Equipo para Aprender Arquitectura de Computadores}

Una vez que a los alumnos se les entrena en las habilidades y actitudes que hacen que el trabajo en equipo funcione y que les permitan diferenciar el trabajo en grupo 
tradicional del trabajo en equipo, se trata de desarrollar las actividades de aprendizaje de las competencias genéricas y específicas de la materia utilizando grupos cooperativos [11].

Para aprender las competencias específicas de la materia se llevan a cabo un conjunto de actividades, cada una de las cuales corresponde a un bloque temático de la asignatura. Para cada actividad hay una parte del trabajo que se realiza de forma individual y otra parte que se realiza en equipo. Realizar una parte del trabajo de forma individual desarrolla la responsabilidad, ingrediente fundamental del aprendizaje cooperativo. Para promover la responsabilidad individual y la interdependencia positiva es necesario que, en las actividades que diseñe el profesor, los alumnos dependan de sus compañeros de equipo, ya sea para ayudarlos o para recibir ayuda. Además, al final de la actividad, se hará una pregunta al azar a cualquiera de los miembros del equipo para asegurar la responsabilidad individual.

Durante las sesiones de teoría y de laboratorio, se ocupa una parte del tiempo para que los grupos realicen parte de la actividad propuesta en horario de clase, incluyendo trabajo individual y en grupo mediante la técnica del puzzle [2], pero además deben dedicar algún tiempo más a trabajar fuera del aula en las diferentes actividades. En concreto, cada actividad ha tenido una duración aproximada de tres semanas. El plan de trabajo para cada actividad, que coincidirá con los temas de la asignatura, se estructura de la siguiente forma:

- Previo a la primera sesión presencial de realización de la actividad, a cada alumno de un grupo se le asigna una actividad diferente, es decir uno realiza un mapa conceptual, otro realiza un glosario de términos, y el otro alumno (en el caso de grupos de tres) preguntas tipo test, y de esta se consigue que lean y trabajen el tema antes de la resolución de problemas.

- En la primera sesión presencial de cada actividad se entrega el resto del trabajo a cada estudiante del grupo cooperativo. Cada actividad está estructurada de forma que hay una parte de trabajo individual diferente para cada miembro del grupo (una cuestión tipo test y un problema diferente para cada alumno del grupo) y una parte común para todo el grupo. Para ello, en dicha sesión presencial, primero individualmente dedican una parte del tiempo a resolver el problema y la cuestión. Pasado ese tiempo se reúne el grupo de expertos (puzzle, alumnos de distintos grupos que tienen asignado el mismo problema) para comparar soluciones y aclarar dudas. Posteriormente, regresan al grupo base original para que cada miembro explique su parte del trabajo al resto de compañeros. La parte grupal de la actividad se realiza fuera de clase y tienen que colaborar todos los miembros del grupo base, teniendo en cuenta los conocimientos adquiridos en la primera parte por cada uno de ellos. Todos tienen que conocer y dominar el trabajo de todo el grupo, ya que pueden ser elegidos aleatoriamente para exponer cualquier parte del trabajo. Finalmente, todo el material se pone antes de la próxima sesión en el Portafolio del grupo (versión sin corregir).

- En la segunda sesión presencial se realiza co-evaluación cíclica (entre los diferentes grupos) del trabajo realizado. La tarea de evaluar a otros grupos forma parte de las competencias transversales de la materia $\mathrm{y}$, por tanto, también será evaluada. Previamente se facilitarán rúbricas para poder realizar correctamente la tarea de evaluación. Una vez realizado dicho trabajo, cada grupo le explica al grupo al que 
ha evaluado qué aspectos se han realizado correctamente y cuáles deben mejorarse y corregirse. La versión corregida del trabajo debe colocarse en el Portafolio en los plazos establecidos, siempre antes de la siguiente sesión.

- En la tercera sesión presencial de la actividad se realiza una presentación oral o una webquest de un trabajo asignado al grupo. Todos los miembros del grupo deben exponer parte del trabajo.

- Por último, cada dos actividades, se realiza una prueba individual de mínimos a todos los alumnos.

Después de la prueba individual dará comienzo una nueva actividad y el ciclo de tres semanas se vuelve a repetir para cada actividad propuesta, intentando además que en la fase de interacción entre los diferentes grupos (puzzle), los alumnos que interaccionan sean distintos.

Es necesario superar los cuatros temas que se evalúan en dos pruebas de mínimos que se realizarán a lo largo del curso. Hay una segunda oportunidad para recuperar cada tema en el examen final.

\subsection{Estructura del Portafolio Digital de Grupo}

El uso del Portafolio digital de grupo ha sido una herramienta más en el proceso de enseñanza-aprendizaje a través de grupos cooperativos. En los últimos años, el Portafolio se ha diseñado haciendo uso de la plataforma digital de apoyo a la docencia WebCT [12]. El último curso (2011-2012) también se ha utilizado la plataforma Moodle, dado que para la misma hemos desarrollado un módulo de Portafolio.

En la plataforma WebCT, a los alumnos se les facilita un archivo html con la estructura básica y los apartados que deben contener todos los Portafolios así como una zona para las fotos y nombres de los integrantes de cada grupo.

El Portafolio se ha organizado en diferentes apartados que han ido dando cuenta de todo el trabajo de aprendizaje y reflexión llevado a cabo por cada grupo. Los apartados definidos para todos los Portafolios han sido los siguientes:

- Objetivos: En este apartado se presentan al estudiante los objetivos que se pretenden alcanzar a través de la realización de las diferentes actividades, así como la importancia del Portafolio para el seguimiento de la asignatura, reflexión del propio trabajo y autoevaluación del grupo.

- Problemas: En este apartado cada grupo debe colocar las diferentes versiones (sin corregir y corregida) de los problemas asignados en cada actividad. Se ofrece una rúbrica sobre los criterios a valorar para la correcta resolución de problemas.

- Glosario. En este apartado se colocan los términos más importantes asociados con cada tema de la asignatura y que será asignado a un alumno diferente del grupo en cada actividad.

- Mapas Conceptuales. En este apartado se coloca el mapa conceptual que es realizado en cada tema por un alumno diferente del grupo. Suelen utilizar herramientas tipo CmapTools para realizarlo e incluirlo en la plataforma. 
- Preguntas test. En este apartado se incluyen las preguntas tipo test a realizar por un alumno diferente del grupo en cada actividad. Suelen utilizar herramientas como el software Hot Potatoes, que permite poder responder a las cuestiones tipo test como si de una prueba o examen se tratara a través de una página web.

- Trabajos. En este apartado los estudiantes colocan los trabajos asignados sobre cada tema, así como cualquier aportación propia que consideren importante en el aprendizaje de la materia.

- Competencias: Este apartado contiene las rúbricas sobre las diferentes competencias a evaluar: trabajo en equipo y comunicación oral. Los estudiantes colocan en primer lugar el reglamento del grupo, que realizan en los primeros días de creación de los grupos cooperativos y que contiene las normas de funcionamiento del grupo así como las autoevaluaciones del mismo.

A modo ilustrativo de lo anteriormente expuesto, en la Figura 1 se muestra la estructura del Portafolio digital que el profesorado le suministra a todos los estudiantes, indicándoles con ello, las secciones que deben tener todos los Portafolios en la asignatura de Arquitectura de Computadores.

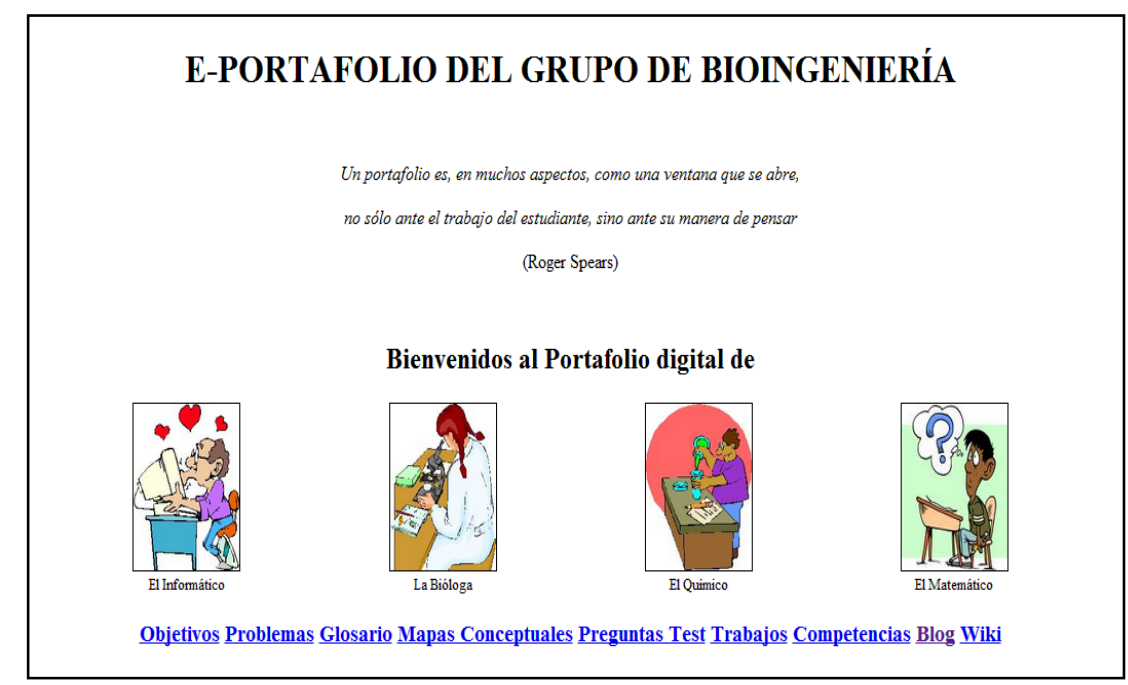

Figura 1. Imagen de la estructura general que todos los Portafolios.

Las Figuras 2, 3 y 4 muestran un ejemplo del resultado final del Portafolio digital de uno de los grupos cooperativos para distintas partes del mismo. En la parte izquierda de la Figura 2 se muestra la estructura del Portafolio que previamente les facilitó el profesorado y que se muestra en la Figura 1 (Objetivos, Problemas, Glosarios, Trabajos, etc). La Figura 3 muestra la parte del Portafolio correspondiente a los problemas que han venido trabajando a lo largo de las actividades durante el curso. Se muestra tanto la versión sin corregir como la versión corregida. La Figura 4 muestra la parte del Portafolio correspondiente a los trabajos, y en concreto uno de ellos que consistía en hacer una Webquest sobre topología de redes estáticas. 


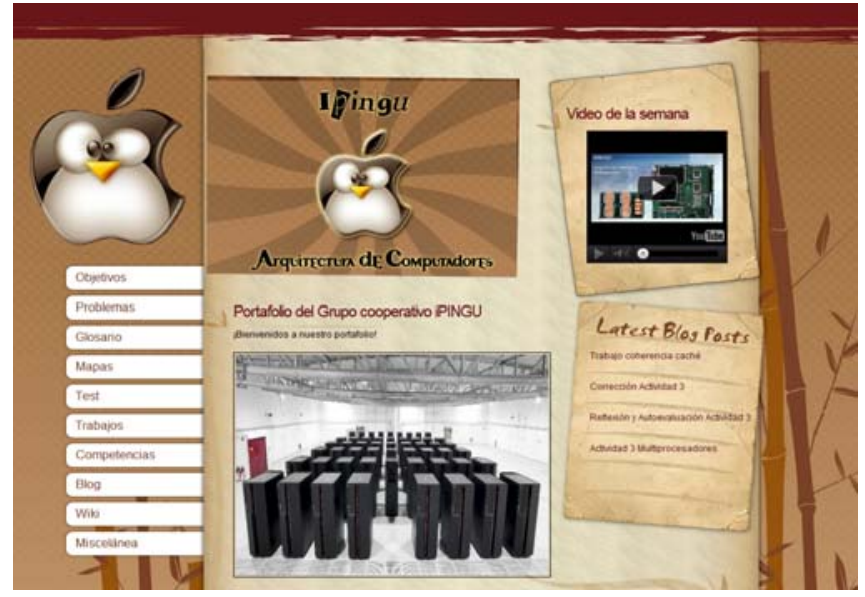

Figura 2. Imagen de la PAGINA INICIAL del Portafolio del grupo Ipingu.

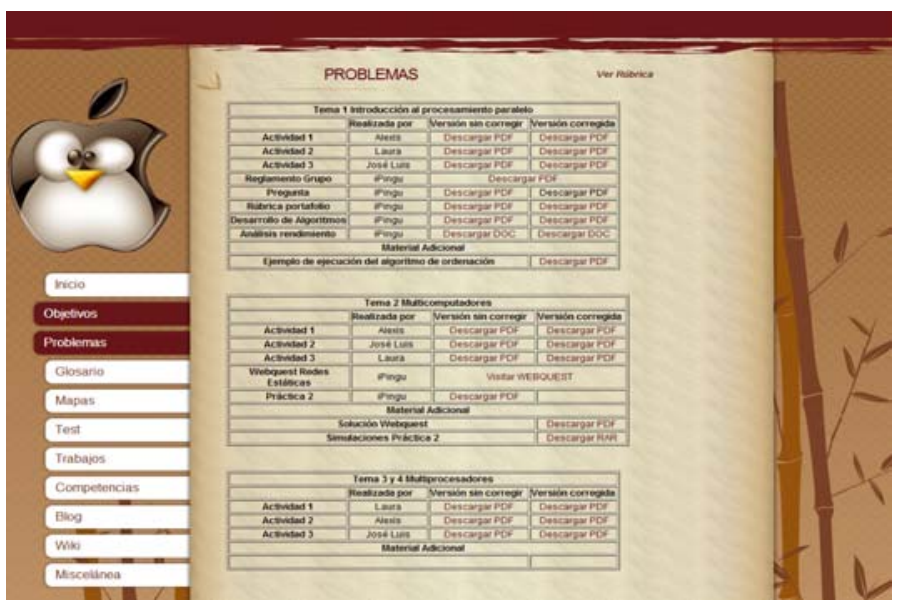

Figura 3. Imagen de la sección PROBLEMAS del Portafolio del grupo Ipingu.

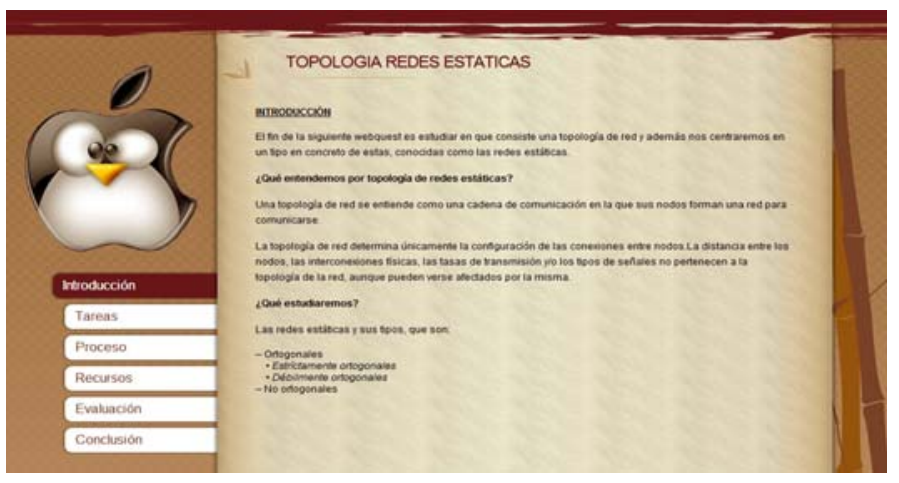

Figura 4. Imagen de la sección de TRABAJOS del Portafolio del grupo Ipingu. 


\section{Evaluación}

Se ha optado por establecer un método de evaluación continua, lo que implica necesariamente un seguimiento y control del avance de cada estudiante a lo largo de la asignatura. Se puntúa de la siguiente forma:

La nota de grupo incluye:

- Las actividades en grupo ponderan un 30\% (glosarios, cuestiones, problemas, prácticas, trabajos, etc.). Se valora un $15 \%$ sólo por entregarlas en la fecha acordada aunque no estén correctas y un 15\% por entregarla corregida en la fecha establecida. Si no se realizan todas las actividades no se superará la asignatura.

- Las preguntas al azar a cualquier miembro del grupo sobre el trabajo presencial realizado en las actividades pondera un $10 \%$ y la nota será para todo el grupo.

- El diseño y desarrollo del Portafolio digital de grupo sobre el que se colocarán todas las actividades tendrá una ponderación del $10 \%$ en la nota.

- La adquisición de competencias suponen un $10 \%$ de la nota, y comprende el trabajo en equipo (5\%), evaluado mediante la asistencia regular al trabajo del grupo en clase, autoevaluaciones de compañeros, observación de la profesora, etc. Y la comunicación oral (5\%), evaluada mediante la presentación de trabajos.

La nota individual incluye:

- Las pruebas individuales ponderarán un $40 \%$ y se valuaran aspectos de conocimientos mínimos de la asignatura. Para superar la asignatura es necesario superar 7 de los 8 conocimientos mínimos evaluados en dos pruebas. La calificación de la componente de conocimientos mínimos variará una vez superados los 7 ó 8 mínimos dependiendo del número de convocatorias utilizadas [7].

\section{Resultados de la experiencia}

Debido a la importancia que para nosotros ha supuesto el uso del Portafolio Digital combinado con los grupos de aprendizaje cooperativo y de cara a una utilización más estándar del mismo, un resultado significativo de esta experiencia, ha sido nuestro interés por desarrollar un módulo Portafolio genérico que se pueda usar en plataformas de libre distribución. Para ello, durante el pasado curso académico 20112012, se ha diseñado y puesto en marcha una nueva herramienta para incluir el Portafolio digital en la plataforma Moodle. Esta plataforma es de libre distribución y hemos empezado a usarla en las asignaturas de Master. Puesto que Moodle permite la creación de grupos de trabajo, lo que se ha hecho es diseñar un módulo que permite al profesor crear el Portafolio de todos los grupos de forma automática a partir de la creación de dichos grupos. Este Portafolio incluye la foto y nombre de los alumnos pertenecientes al grupo, un foro accesible únicamente para los miembros del grupo, secciones para organizar el trabajo de los estudiantes, y un área para manejar archivos. A diferencia del Portafolio que hemos utilizado en WebCT, donde son los alumnos los que realizan todo el diseño del portafolio en ficheros HTML (Figuras 2, 3 
y 4), a través de este nuevo módulo, hemos conseguido descargar a los alumnos de esa tarea, ya que ahora, en Moodle es el sistema el que lo genera automáticamente, con el consiguiente ahorro de tiempo para los estudiantes (Figuras 5, 6, y 7). El diseño de todos los Portafolios en Moodle es el mismo y sólo varían las secciones personales que pueden incluir los estudiantes.

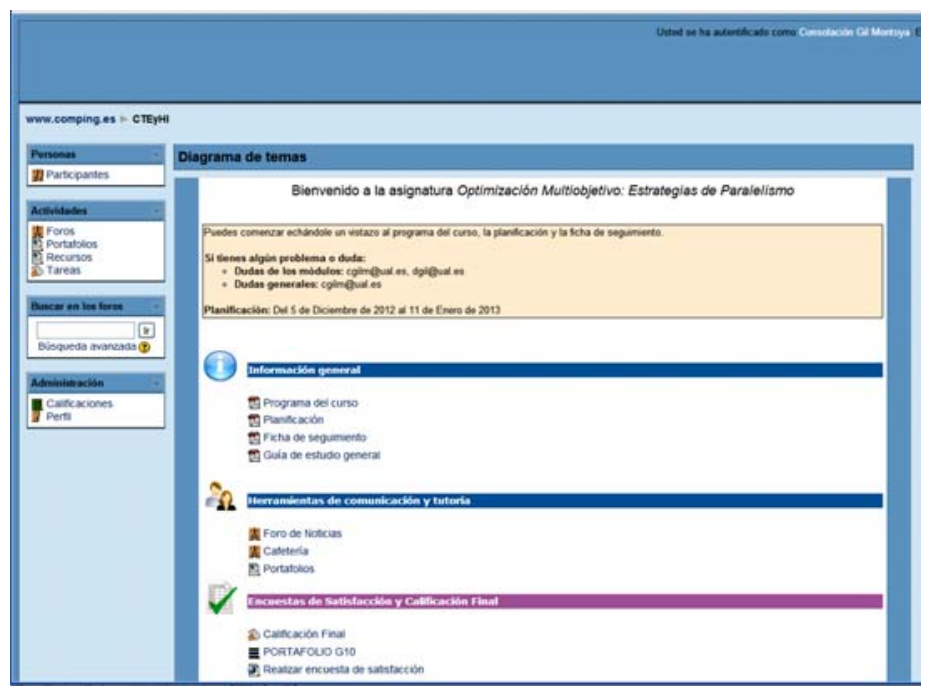

Figura 5. Plataforma Moodle para la asignatura OMEP del Master TIA.

La Figura 5 muestra la página inicial de la asignatura Optimización Multiobjetivo: Estrategias de Paralelismo (OMEP) del Master Técnicas Informáticas Avanzadas (TIA). En la parte izquierda de dicha figura se observa el acceso al Portafolio.

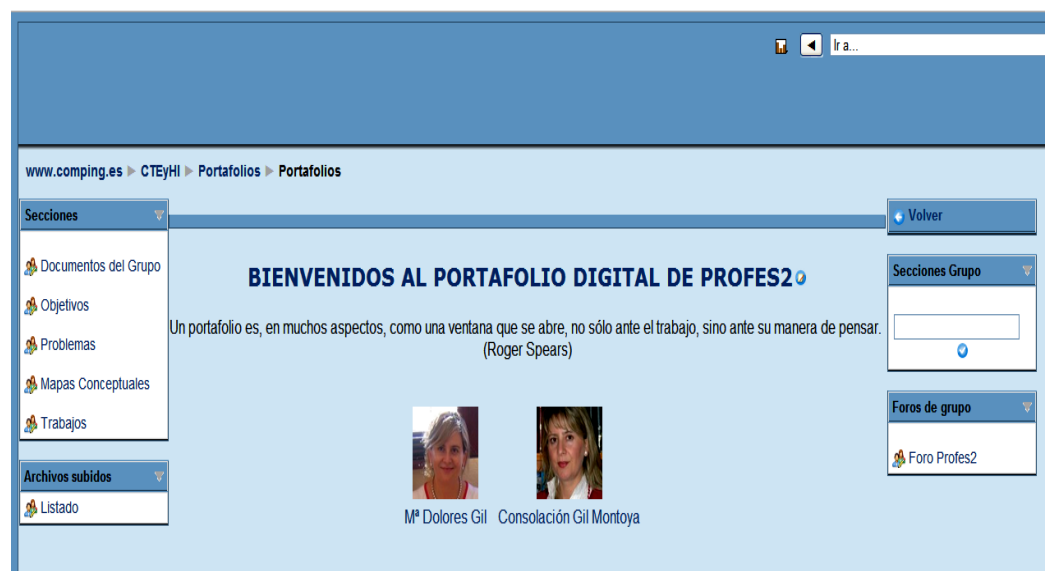

Figura 6. Portafolio del grupo cooperativo de las profesoras. 
La Figura 6 muestra la imagen del Portafolio. En este caso se ha optado por poner el Portafolio de las profesoras, para evitar poner la foto de los estudiantes. En la parte izquierda aparecen las secciones que las profesoras han diseñado para todos los grupos, además del manejo de archivos. En la parte derecha se encuentran las secciones de los alumnos y el acceso al foro específico del grupo.

Por último, la Figura 7 muestra el acceso a una de las secciones de trabajo del grupo, en este caso, la parte que muestra el reglamento del grupo.

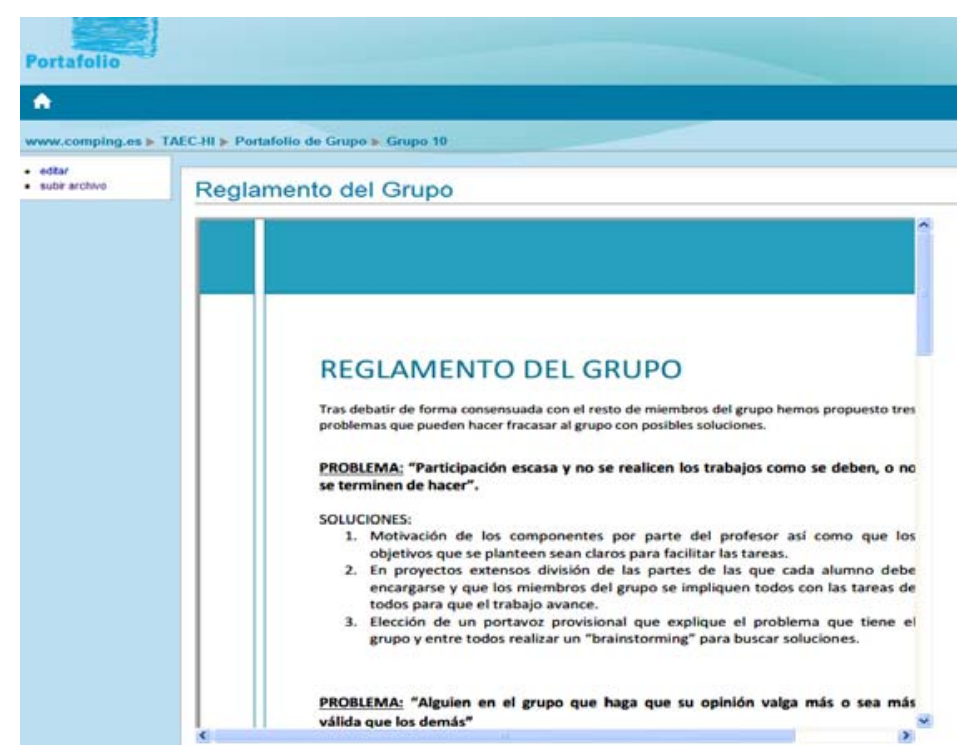

Figura 7. Imagen de la sección de TRABAJOS del Portafolio digital de un grupo.

Los resultados obtenidos a lo largo del tiempo denotan claramente que el número de alumnos que de forma voluntaria optan por esta metodología, en detrimento de jugárselo todo en el examen final, se ha ido incrementando progresivamente. Esto denota que los estudiantes están más motivados por la asignatura, discuten, hablan, preguntan y algo fundamental para el profesorado, es que siguen la asignatura al día, sin acumular la materia para estudiar en los días previos al examen final.

Los datos concretos, presentados en la Figura 8 muestran como, por un lado, el porcentaje de estudiantes que, en lugar de desistir en preparar la asignatura, la preparan y se presentan a los exámenes aumenta considerablemente desde el momento en el que se empiezan a aplicar estas metodologías activas. De igual forma, el número de aprobados también ha sido bastante sorprendente, tal y como muestra la Figura 8, ya que se ha pasado de un $60 \%$ de aprobados en el sistema tradicional a un porcentaje que ronda el $90 \%$. 


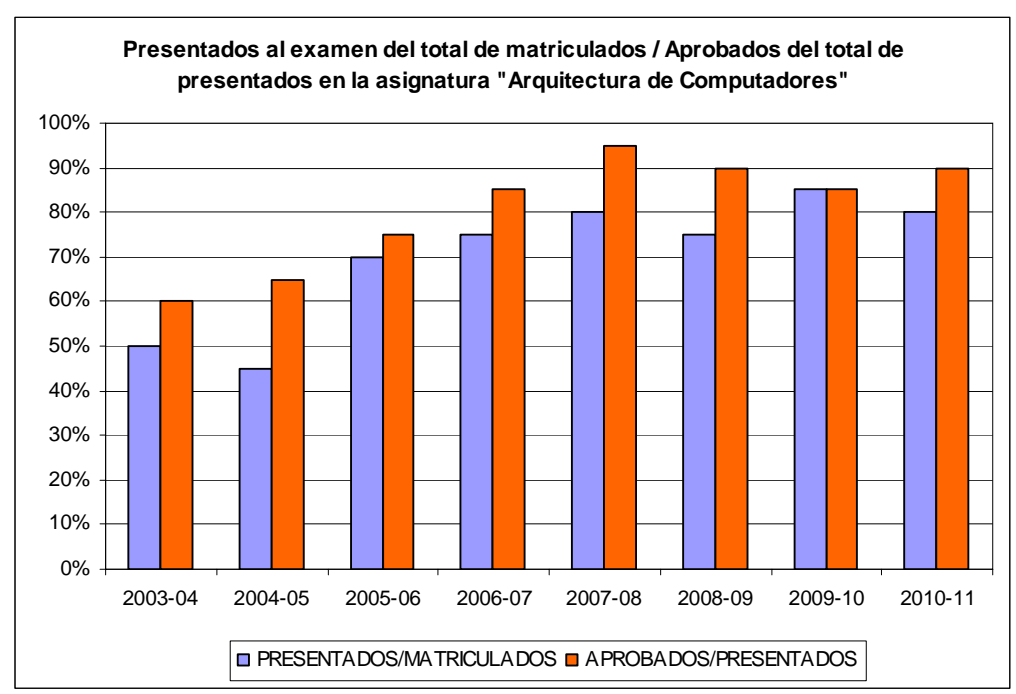

Figura 8. Gráfica sobre alumnos presentados a examen y sobre los aprobados desde el curso 2003-04. Estas metodologías se introdujeron en el curso 2005-2006.

\section{Conclusiones}

El aprendizaje cooperativo le permite al docente alcanzar varias metas importantes al mismo tiempo. En primer lugar, le ayuda a elevar el rendimiento de todos sus alumnos, incluidos tanto los especialmente dotados como los que tienen dificultades para aprender. En segundo lugar, lo ayuda a establecer relaciones positivas entre los alumnos, sentando así las bases de una comunidad e aprendizaje en la que se valore la diversidad. En tercer lugar, les proporciona a los alumnos las experiencias que necesitan para lograr un desarrollo social, psicológico y cognitivo. La posibilidad que brinda el aprendizaje cooperativo de abordar estos tres frentes al mismo tiempo lo hacen superior a todos los demás métodos de enseñanza. El uso de grupos de aprendizaje cooperativo ha mejorado no sólo los resultados académicos sino también la motivación hacia estas asignaturas, que de partida, encuentran más difíciles.

Uno de los puntos fuertes del Portafolio digital es la retroalimentación externa que el estudiante recibe continuamente de su trabajo, no sólo por parte del profesorado, sino por sus propios compañeros. Ello hace que el grupo reflexione sobre sus propios trabajos, su proceso de aprendizaje, sus resultados y sus conclusiones.

Los resultados obtenidos muestran que los estudiantes están más motivados y como consecuencia se implican activamente en las diferentes actividades propuestas, además de conseguir involucrar a un mayor número de alumnos en el seguimiento diario de la asignatura. Todo ello llevado al campo de los resultados evaluables, se ha traducido en un mayor número de presentados y también aprobados en la asignatura, así como en una mayor satisfacción, no sólo para los alumnos, sino también para los docentes que han impartido la materia. 


\section{Referencias}

1. Panitz, T. (1999) Benefits of cooperative learning in relation to student motivation. En Michael The-all (Ed.). Motivation from within: Approaches for encouraging faculty and students to excel, New directions for Teaching and Learning, 78. San Francisco, CA: Josey-Bass publishers.

2. Johnson, D.W, Johnson, R.T. Holubec, E.J. (1999) El aprendizaje cooperativo en el aula. Ed. Paidos.

3. Klenowski, V. (2004) Desarrollo de portafolio. Para el aprendizaje y la Evaluación. Madrid: Narcea.

4. Gibbs, G. (2003) Uso estratégico de la educación en el aprendizaje. En Brown, S. y Glasner, A. (Ed.). Evaluar en la Universidad. Problemas y nuevos enfoques (pp. 61-75). Madrid: Narcea.

5. Barberà, E, Bautista, G.; Espasa, A., Guasch, T. (2006) Portafolio electrónico: desarrollo de competencias profesionales en la red. Revista de Universidad y Sociedad del Conocimiento, 3(2), pp. 55-66.

6. Johnson, D.W., Johnson, R.T. (1989). Cooperation and competitions. Theory and research. Edina, MN. Interaction Book Company.

7. Valero, M., Diaz de Cerio, L. (2005) Autoevaluación y co-evaluación: estrategias para facilitar la evaluación continuada. Actas del I Simposio Nacional de Docencia en la Informática (pp. 25-32), Granada, Septiembre.

8. Putnam, J. (1997) Cooperative learning in diverse classrooms. Upper Saddle River. N.J: Prentice-Hall.

9. Pujolàs, P. (2005) El cómo, el porqué y el para qué del aprendizaje cooperativo. Cuadernos de Pedagogía, 345, pp. 51-54.

10. Fabra, M.L. (1994). Técnicas de grupo para la cooperación. Barcelona: CEAC.

11. Villa, A., Poblete, M. (Dirs.) (2007) Aprendizaje basado en competencias. Una propuesta para la evaluación de competencias genéricas. Bilbao: Mensajero/ICE Universidad de Deusto.

12. Gil, C., Montoya, M.G., Herrada, R.I., Baños, R., Márquez, A.L., Alcayde, A. (2012) Capítulo 7: Prácticas de aprendizaje cooperativo y del portafolio digital de grupo: Aprender a cooperar y cooperar para Aprender, En M.F. Serrano (Coord). Aprendizaje Cooperativo en Contextos Universitarios, pp: 123-154. Editorial Universidad de Murcia. 